\title{
Neue Lernformen, flexible Zeiten: Der Zukunftstrend bei dentaler Fortbildung
}

\author{
Der APW-Vorsitzende Dr. Norbert Grosse blickt anlässlich des 5000. APW-Mitglieds voraus
}

I

m Jahr eins nach ihrem 40jährigen Bestehen setzt die Akade1 mie Praxis und Wissenschaft (APW) als Tochter der DGZMK erneut ein Zeichen auf dem dentalen Fort- und Weiterbildungsmarkt. Mit Dr. Britta Hansmeier aus Künzelsau konnte die APW unlängst ihr 5000. Mitglied begrüßen. Dazu wird man, indem man ein Curriculum bei der APW erfolgreich abschließt. Was einst mit der Serie „Allgemeine Zahnheilkunde“ ganz klein begann, hat im Lauf der Jahrzehnte die postgraduale Schulung im Bereich der Zahnmedizin maßgeblich mitgeprägt. Im Interview schildert der APW-Vorsitzende Dr. Norbert Grosse (Wiesbaden), warum es keinen Anlass gibt, sich auf Lorbeeren auszuruhen und wo die Herausforderungen der zahnmedizinischen Fortbildung in Zukunft liegen.

djz: Was hat die APW in der Vergangenheit so erfolgreich gemacht? Dr. Norbert Grosse: Zu Anfang war die APW zweifellos ein Trendsetter im dentalen Fortbildungsmarkt und nahezu konkurrenzlos. Das hat sich im Lauf der Zeit gründlich geändert. Viele andere Anbieter sind auf den Markt gedrängt, die sich hier viel stärker engagieren als früher. Aber die APW konnte

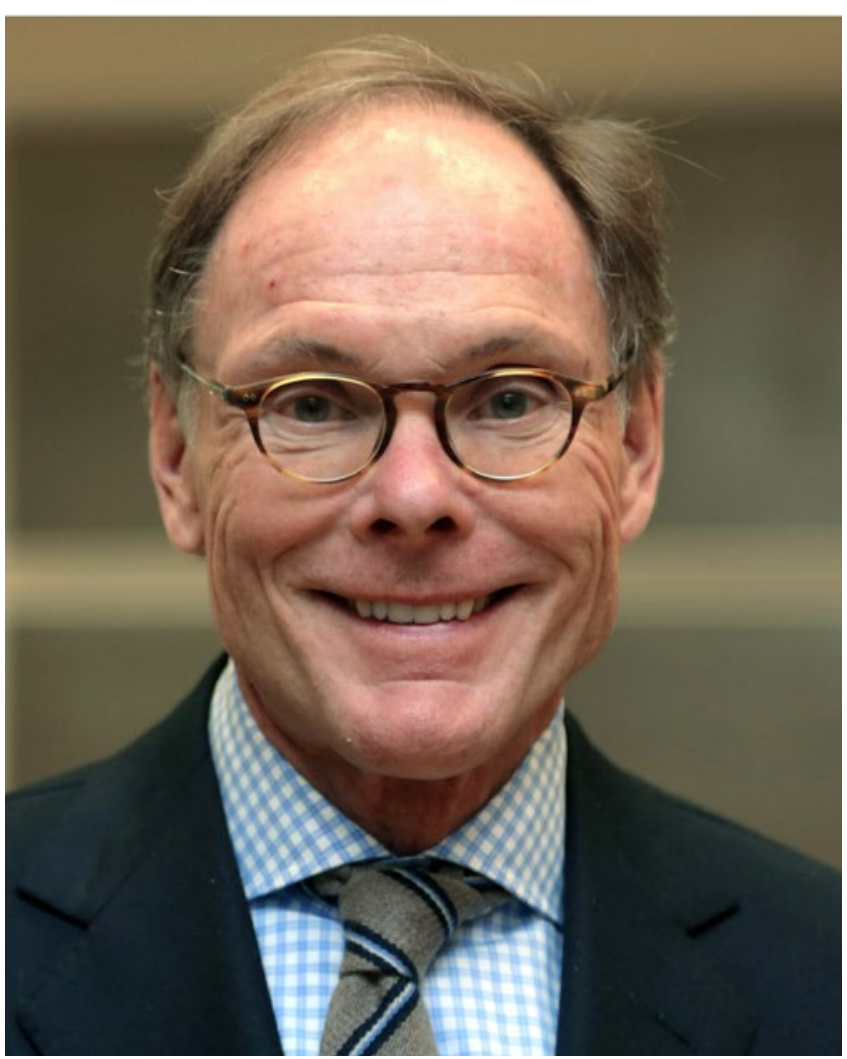

Dr. Norberte Grosse sieht die APW für die Zukunft gut gerüstet sich dadurch behaupten, dass sie sich mit ihren Angeboten den gewandelten Anforderungen der Teilnehmerinnen und Teilnehmer angepasst hat. So haben wir in der jüngeren Vergangenheit auch mit neuen Veranstaltungsformen wie etwa 'APW select' und einer stetigen Ausweitung der curriculären Angebote überzeugt. Zur Zeit laufen 25 Serien mit 13 verschiedenen Fachgebieten in der APW parallel, insgesamt organisieren wir im Jahr zwischen 5000 und 6000 Kursplätze.

djz: Die Anforderungen an einen Fort- und Weiterbildungsanbieter haben sich gewandelt. Wo sehen Sie für die Zukunft die größten Herausforderungen?

Grosse: Das ist richtig. Mit der Entwicklung des Fachs und dem Trend zu Spezialisierungen, aber darüber hinaus auch mit dem gewandelten Berufsbild und der wachsenden Feminisierung des Berufsstandes, haben sich die Erwartungen geändert. So möchten immer mehr Frauen in unserem Beruf ihre Fortbildungszeiten und die familiären Verpflichtungen in Einklang bringen. Die veränderten Kommunikationsformen und -gewohnheiten durch den Siegeszug der IT bergen auch neue Formen des webbasierten Lernens, die wir uns zunutze machen wollen. Lernen überall und zu jeder Zeit via PC, Smartphone oder Tablet wird zur Selbstverständlichkeit werden. Wobei die Unterstützung unserer Präsenzveranstaltungen mit digitalen Medien in virtuellen Kursräumen einen ersten Schritt darstellt. Darauf stellen wir uns auch mit dem neuen Internet-Portal owidi ein, das uns ganz neue Möglichkeiten bietet und dessen bundesweites Rollout in diesen Tagen beginnt.

djz: Die Technik in der Wissensvermittlung allein dürfte aber kein Garant für Erfolg sein. Was ist mit dem APW-Angebot selbst?

Grosse: Wir können uns von der Konkurrenz im dentalen Fortbildungsmarkt nur absetzen, wenn wir die richtigen und gewünschten Fortbildungsinhalte in geeigneten Formaten und mit entsprechend hochklassigen Referenten anbieten. Hier gilt es, den richtigen Riecher zu haben. Auch zwischen den Präsenzveranstaltungen und dem Online-Lernen muss die richtige Balance gefunden werden, denn viele Kolleginnen und Kollegen sind nicht als 'digital natives' auf die Welt gekommen. Durch die Erkenntnis, dass es sehr unterschiedliche Lernpräferenzen gibt, wird man sich auf neue Lernprozesse und Lerndidaktiken aber genauso einstellen müssen wie auf ein verändertes Lernmanagement der Teilnehmer. Diese Möglichkeiten der digitalen Welt umsetzen zu können, ist von der organisatorischen Seite zunächst nur mit hohem personellen Engagement und erheblichen Investitionen möglich. Mit den besonderen Chancen, die die Ankopplung der APW an das Lernportal owidi uns bietet, sehe ich die APW für die Zukunft gut gerüstet!

Das Interview führte Markus Brakel 